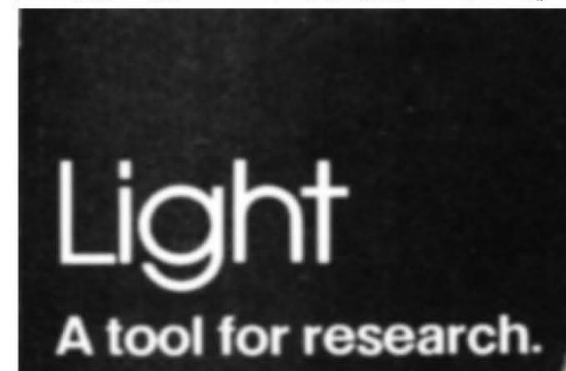

SLM's "Smart" 8000 Photon-

Counting Spectrofluoro-

meter harnesses the

power of light to help

you learn more

about our world

using

fluorescence

spectroscopy.

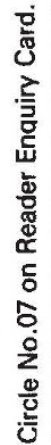

devoted to the causal analysis of morphogenesis and morphogenetic models. In this area the literature of regeneration is not noted for its objectivity. Wallace, again, makes no secret of his own personal bias, but provides a thoughtful analysis of the experimental evidence underlying most of the current models. The last chapter contains speculative material that summarizes a number of the author's own ideas on regeneration, a major one being that limb regeneration is strictly a repetition of limb development.
Whether or not one agrees with some of the arguments and conclusions, this book is valuable not only for the amount of information it contains, but also for the original thoughts and interpretations. It should meet the author's expectations of providing a stimulus for future investigation into some of the outstanding issues of limb regeneration.

Bruce M. Carlson is a Professor in the Departments of Anatomy and Biological Sciences at the University of Michigan.

\title{
Academic barriers at high altitude
}

\section{Peter D. Moore}

Ecology of Highlands. Monographiae Biologicae, Vol.40. By M.S. Mani and L.E. Giddings. Pp.247. ISBN 90-6193-093-6. (Dr W. Junk, The Hague: 1981.) Dfl.110, $\$ 58$.

EXTREME environments, such as the deep seas and very high altitudes, present peculiar ecological problems to the plants and animals which inhabit them. Few books have been written on the deep seas, whereas many have been written about the ecology, flora and fauna of the high mountains. But most of these go little further than to describe the unusual features of "alpine" plants and animals inhabiting those regions, often in terms of their morphology, reproductive strategies or biogeographical significance. This book attempts to go further by concentrating upon the physical environment of high altitude habitats, the effects that extreme physical conditions have upon plants and animals (including human beings) and the adaptation resulting from the evolutionary responses of these organisms.

The twin authorship reflects the two aspects of the problem. L.E. Giddings (Veracruz, Mexico) is responsible for the sections on the physics and meteorology of the habitat and M.S. Mani (Agra, India) covers the biological aspects. Such a dual approach is novel and brave, the main problem being the integration of the two sections, especially in view of the disciplinary and geographical gulf between the two authors.

Introductions are places for generalizations, which may be modified and expanded later in the text. The introduction by Mani, however, contains generalizations which have a rather inflexible ring to them. One would expect some overall definition of highland in terms of altitude. Instead, the definition concentrates upon low atmospheric pressure, cold and aridity (the latter surely being very variable). Then we are given such dogmatic statements as "The altitude at which the mean annual air temperature in the shade does not rise above $10^{\circ} \mathrm{C}$ is the timberline"'. No indication is given that this might vary with such factors as wind. Indeed, the essentially continental (in a meteorological sense) experience of the authors in the mountains of Asia and South America is evident in the book, which will limit its value to those working in oceanic areas.

We then have eight chapters by Giddings on the physical environment of high altitudes. There are many useful and interesting sections in this part of the book, though it does suffer from a surfeit of tabulated data relating to atmospheric variables, which could have been relegated to appendices.There is also a strong emphasis upon instrumentation and upon engineering and physical features of the environment which have a very flimsy connection with its ecology. (Does the ecologist really need to know how long it will take him to boil an egg at high altitude?) Indeed, there is no systematic attempt to relate the physics to the biological problems discussed later by Mani.

Mani begins with general surveys of plant and animal life at high altitude, much of which is a condensation of his previous books on highland insects and plants. There is a strong Himalayan flavour to these chapters, but data from other areas are incorporated. The taxonomic arrangement of material is surprising and this does little to ease the transfer of authorship and emphasis, though the animal chapter does attempt to relate adaptation to physical factors.

The final chapters concentrate on human beings, especially the physiological peculiarities found in high altitude peoples.

Overall the book does not succeed in integrating the two approaches to the highland environment except in rather isolated sections of Mani's accounts of animal and human adaptation. It was an ambitious idea but, sadly, the barriers of longindependent development within the two scientific disciplines, coupled perhaps with the geographical isolation of the authors, have proved too great.

Peter D. Moore is Senior Lecturer in the Department of Plant Sciences at King's College, University of London. 